\title{
Circulating Tumor Cells: Liquid Biopsy for Early Detection of Cancer
}

\author{
Benjamin Mwesige ${ }^{1}$, Seung-Gu Yeo ${ }^{2, *}$, Byong Chul Yoo ${ }^{1,3, *}$ \\ 'Department of Cancer Biomedical Science, Graduate School of Cancer Science and Policy, National Cancer Center, Goyang; ${ }^{2}$ Department of Radiation \\ Oncology, Soonchunhyang University Cheonan Hospital, Soonchunhyang University College of Medicine, Cheonan; ${ }^{3}$ Biomarker Branch, Research Institute, \\ National Cancer Center, Goyang, Korea
}

\begin{abstract}
Cancer is a complex, heterogeneic, and dynamic disease involving multiple gene-environment interactions, and affecting numerous biological pathways. As such, the development of reliable and robust non-invasive platforms constitutes a vital step toward realizing the potential of precision medicine. Distant metastases harbor unique genomic characteristics that are not detectable in the corresponding primary tumor of the same patient, and metastases located at different sites show considerable intra-patient heterogeneity. Thus, the analysis of the resected primary tumor alone or, if possible, re-evaluation of tumor characteristics based on the biopsy of the most accessible metastasis, may not reveal sufficient information for treatment decisions. Here, we propose that this dilemma can be solved by a new diagnostic concept: liquid biopsy, that is, the analysis of therapeutic targets and drug resistance-conferring gene mutations in or on circulating tumor cells (CTCS). Finally, the analysis of the resected primary tumor alone may provide misleading information with regard to the characteristics of metastases, the key target for systemic anticancer therapy. Liquid biopsies are noninvasive tests using blood or fluids that detect CTCs or the products of tumors, such as fragments of nucleotides or proteins that are shed into biological fluids from the primary or metastatic tumors. Such biopsies are expected to be informative or easily accessible tools to provide comprehensive information regarding cancers beyond conventional biopsies. Thus, this review addresses the use of CTCs in cancer detection, diagnosis and monitoring and discusses the direction of its clinical application in cancer patient care.
\end{abstract}

Keywords: Circulating neoplastic cells; Liquid biopsy; Precision medicine

\section{INTRODUCTION}

Cancer-specific mortality from most types of solid tumors has barely decreased in decades despite an exponential increase in our knowledge about cancer pathogenesis and significant investments in the development of effective treatments. The past few years have witnessed the dramatic success of immunotherapies in treating a subgroup of patients with a variety of tumor types, including lung, bladder, and kidney cancers, as well as Hodgkin's lymphoma and melanoma. While such breakthroughs offer the hope of prolonged survival for some patients with advanced cancers, finding cancers earlier would still afford the greatest chance for a cure, given that the survival rates for patients with early diagnoses are 5 to 10 times higher compared with late-stage disease [1]. By enabling the diagnosis and localized treatment of early-stage invasive cancers and, in some cases, pre-invasive states, screening for cervical and colorectal cancers has contributed to significant declines in mortality from these diseases. Similarly, effective population screening paradigms for many other common and deadly tumor types are urgently needed to reduce cancer-specific mortality. Currently, physicians and researchers work toward providing accurate cancer screening, diagnosis, and prognostication along with a correct prediction of recurrence and resistance to treatment [2]. Traditionally, physicians and researchers have regarded tissue biopsy as

Correspondence to: Byong Chul Yoo

Biomarker Branch, Research Institute, National Cancer Center, 323 Ilsan-ro, Ilsandong-gu, Goyang 10408, Korea

Tel: +82-31-920-2342, Fax: +82-31-920-2354, E-mail: yoo_akh@ncc.re.kr

(C) 2019 Soonchunhyang Medical Research Institute This is an Open Access article distributed under the terms of the Creative Commons Attribution Non-Commercial License (http://creativecommons.org/licenses/by-nc/4.0/).

Co-correspondence to: Seung-Gu Yeo

Department of Radiation Oncology, Soonchunhyang University Cheonan Hospital, 31 Suncheonhyang 6-gil, Dongnam-gu,

Cheonan 31151, Korea

Tel: +82-41-570-3557, Fax: +82-41-592-3809, E-mail: md6630@schmc.ac.kr

*These two corresponding authors contributed equally to this work.

Received: Dec. 26, 2018 / Accepted after revision: May 18, 2019 
the gold standard for providing data that produce positive health outcomes among patients with a variety of cancers. Furthermore, tissue biopsy provides the histopathological information that helps physicians determine whether a lesion is malignant [3]. However, it is worth noting that commonly used tissue biopsy methods, such as surgical biopsy, are invasive, and their effectiveness depends on the cancer stage and local area that is biopsied [3]. Having established that there is considerable tumor heterogeneity, taking multiple biopsies from the patients' primary tumor and metastases would seem to be the most obvious next step; therefore, why is it not routinely done? There are many difficulties involved in obtaining a tissue biopsy, including the discomfort suffered by the patient, inherent clinical risks to the patient, potential surgical complications, and economic considerations, meaning that multiple or serial biopsies are often impractical. In addition, some tumors are not accessible for biopsy; the procedure itself might increase the risk of the cancer 'seeding' to other sites [4] and the procedure might not be recommended for patients receiving anti-angiogenic treatment [5].

Even in an ideal situation, where several metastatic sites can be biopsied simultaneously, the analysis of samples can delay the initiation of treatment and may irremediably jeopardize it. These limitations are particular restraints in the context of acquired resistance to therapy, as the ability of a clinician to detect therapeutic biomarkers at an early stage would allow for a potentially successful change in the treatment course. The conventional gold standard of using single biopsies of the primary tumor to inform all downstream therapeutic decisions may no longer be adequate given the increasing evidence of significant spatial and temporal heterogeneity in tumors [6], especially when placed under the selection pressure of various treatments. Moreover, serial biopsies are often impractical and technically challenging. Some tumor entities, such as lung cancer, are located at remote sites and a needle biopsy can be very difficult and high-risk. Finally, the analysis of the resected primary tumor alone (current standard practice in oncology) may provide misleading information with regard to the characteristics of metastases, the key target for systemic anticancer therapy [5]. Distant metastases can harbor unique genomic characteristics that are not detectable in the corresponding primary tumor of the same patient, and metastases located at different sites show considerable intra-patient heterogeneity. Thus, even if reevaluation of tumor characteristics based on the biopsy of the most accessible metastasis is possible, it may not reveal sufficient information for treatment decisions [7]. As a matter of fact, there is growing evidence for intra-tumor heterogeneity indicating that single-site biopsies fall short of revealing the complete genomic landscape of a tumor. Precision medicine and personalized medicine are based on the development of biomarkers, and liquid biopsy is able to detect biomarkers that carry information on tumor development and progression. Compared with traditional 'solid biopsy', which cannot always be performed to determine tumor dynamics, liquid biopsy has notable advantages in that it is a noninvasive modality that provides diagnostic and prognostic information prior to treatment, during treatment and during progression. In this review, we describe the source, characteristics, technology for detection, and current situation of circulating tumor cells (CTCs), circulating-free DNA, and exosomes used for diagnosis, recurrence monitoring, prognosis assessing, and medication planning [8].

Extensively altered cells do not respond to regulatory stimuli and tend to form lumps, leading to malignant tumors that can either be confined to the related organ or begin spreading by invading the basement membrane and/or the formation of new blood vessels. Cancer is very heterogeneous and variability is often seen among different ethnicities and races, although individuals from different regions may harbor the same tumor [9]. The process of spreading from the primary tumor site to proximate or distant sites is considered one of the most threatening aspects of cancer [8]. It is the primary cause of treatment failure and cancer-related death. Cancer cells tend to invade secondary sites (i.e., bone, liver, lung, and brain) and exfoliate into body cavities, especially the pleural space, where they grow in suspension within effusions [10]. Metastatic cells circulate in the bloodstream even after trials to eradicate the primary tumor and often remain undetectable at diagnosis. These CTCs, which are suspected to be the origin of metastatic disease, can easily be found in the peripheral blood and bone marrow of cancer patients.

\section{CIRCULATING TUMOR CELLS}

The first observation of tumor cells in blood was made by Thomas Ashworth in 1869 [11]. In subsequent reports, CTCs were only observed in blood when present in high numbers. As technology advanced, it became possible to detect the presence of CTCs at a much lower concentration. For example, various polymerase chain reaction techniques can be used to detect CTCs in 
blood but are less suitable for enumeration [11]. The combination of flow cytometry with a magnetic enrichment step allowed for CTC enumeration down to 1 cell per mL. CTCs in peripheral blood of patients with metastatic disease are very rare and range from 0 to 10,000 CTCs per $\mathrm{mL}$ of whole blood [12]. Prospective clinical studies in breast, colon, and prostate cancers have shown that the presence of CTCs in $7.5 \mathrm{~mL}$ of blood strongly correlates with progression-free survival and overall survival (OS) [13]. The blood samples were taken after patients received the first cycle of chemotherapy. Intuitively, the difference between $<1$ CTC and 1-4 CTCs should make a larger difference, but because the complete blood volume is measured, the concentration of CTCs contains a poison error due to sampling. This observation together with the chance of a false-positive makes it difficult to distinguish between none and a few CTCs. If error-free detection is possible, even one CTC in $7.5 \mathrm{~mL}$ would already lead to a worse prognosis [13]. Lower concentrations of CTCs can be found in patients when the analysis of a higher volume is possible. If the concentration of CTCs currently found in patients is plotted as a cumulative distribution function, a distribution can be plotted to extrapolate the lower concentrations [14]. The definition of a CTC also has a strong influence on this threshold. For example, in immunofluorescent microscopy, a more loose definition of what a CTC is could result in higher counts in patients and in healthy controls but also in a lower distinguishing power [15]. It is important to select only the CTCs or objects that influence the outcome. Comparisons between CTCs and other predictors of prognosis or response to therapy, such as serum tumor markers and imaging modalities, have shown that CTCs perform well and are independent predictors of outcome.

\section{CLINICAL APPLICATION OF CIRCULATING TUMOR CELLS}

Molecular characterizations of CTCs have the potential to play multiple roles in the pathogenesis process, including being used as biomarkers or surrogates for OS prognosis, staging, biomarker discovery, and personalized treatment, by serving as 'liquid biopsies' [16]. However, tumor tissue biopsy is an invasive procedure with several limitations in clinical practice: it often provides insufficient materials; it constitutes a single snapshot of the tumor, and is subject to selection bias resulting from tumor heterogeneity. Moreover, tumor tissue specimens cannot be easily obtained re- peatedly to track tumor dynamics over time because most diagnostic procedures are invasive and not acceptable for patients. Liquid biopsy has been widely used a viable surrogate for tumor tissue, for the noninvasive assessment of tumor-specific biomarkers and can be used for a variety of clinical and investigational applications [16]. Indeed, different tumor-derived components can be isolated from blood, including circulating cell-free tumor DNA (ctDNA), cell-free tumor RNA, exosomes, tumor-educated platelets, and CTCs, and can be leveraged to uncover the molecular landscape of the tumor and capture the heterogeneity of metastatic cancers [17]. Different body fluids (other than blood), such as urine, cerebrospinal fluid, saliva, and pleural effusions, can be a source of tumor-derived molecular information. In breast cancer, CTCs have been explored successfully as a surrogate for human epidermal growth factor receptor 2 (HER2) expression/amplification [18], and alterations in CTC count may aid in determining the sensitivity or resistance to various cancer treatments [19]. In prostate cancer, prostate-specific antigen (PSA) levels are weakly associated with better survival and thus are not sufficient to guide treatment in the first trimester [20]. In a few cases of highly advanced and androgen receptor-driven stages, PSA fails to be reliable. Hence, bone scans are required every 6 weeks to avoid any relapse in response to treatment. To overcome these limitations, the Veridex CellSearch System, a Food and Drug Administration (FDA)-cleared assay for the enumeration of CTCs [21], was approved based on several studies carried out on breast [22], prostate [23], and colorectal [23] cancers. Accurate and repeated biopsies of tumor tissues are crucial for an improved understanding and monitoring of changes in malignant cell populations during disease progression and in response to therapies. Attempts are being made to replace invasive tissue biopsies for monitoring by socalled liquid biopsies, which can be easily and frequently repeated and rely on the detection of either circulating DNA or cancer cells. Thus, the detection and characterization of CTCs offer the potential to estimate the risk of metastatic relapse, to stratify patients according to different adjuvant therapies, to identify new therapeutic targets, and to monitor systemic anticancer therapies [24].

Clinically, patients with metastatic disease are often characterized by the presence of one or more micro- and macrometastatic foci throughout various organs [25]. Only some of these foci are clinically detectable at the time of metastasis diagnosis, while many more foci are likely to be present but below the detection limit, posing a significant risk in terms of disease progression [26]. 
In this context, treatment strategies for patients with metastasis are often based on the pathological and molecular characterization of the primary tumor, while little or no information is available from the various metastatic lesions present at that specific moment. One main reason for this is that metastases are hardly accessible for direct sampling. However, the consequence is that we still treat metastases based on information obtained from the primary tumor, an approach that has led to scant success. Furthermore, we are now aware of the fact that metastasis is an evolutionary process, where metastatic sub-clones with a unique mutational profile may emerge along with cancer progression at any time, resulting in a high degree of heterogeneity and significant differences from the primary tumor [27]. Technically, our current understanding of cancer heterogeneity, especially in the metastatic setting, indicates that even if we were able to biopsy most of the metastatic lesions in a given patient, we would still face the issue of cellular heterogeneity within each lesion [28]. However, newly established protocols now enable a single-cell resolution interrogation of the genome and transcriptome of cancer cells, and their application to the metastasis field holds great promise for defining its molecular drivers with high precision [29].

While metastasis remains very challenging for a direct biopsy, recent developments in microfluidics technologies are enabling the capture of live CTCs from the blood of patients with various types of cancer [30]. Most excitingly, given the short half-life of CTCs in circulation, in the metastatic setting, CTCs are a direct derivative of metastasis [31]. Pragmatically, they can be seen as an opportunity to isolate, in real time, live cancer cells that are derived from proliferating metastatic lesions in patients, thereby empowering a single-cell resolution analysis of metastasis from a minimally invasive liquid biopsy. CTC enumeration and characterization with certified systems provides reliable information on prognosis and may serve as a liquid biopsy to identify therapeutic targets or the mechanism of resistance in metastatic cells, such as mutations in KRAS or the expression of androgen receptor variant 7 [32]. Metastatic cells might have unique characteristics that differ from the bulk of cancer cells in the primary tumor currently used for the stratification of patients to systemic therapy. Moreover, monitoring of CTCs before, during and after systemic therapy (for example chemotherapy, hormonal therapy, and antibody therapy) might provide unique information for the future clinical management of the individual cancer patient and serve as a surrogate marker for response to therapy [33].
The emergence of increasingly advanced and sensitive technologies to isolate human CTCs provides the opportunity to extend studies of cancer metastasis directly to human cancer. The full range of potential applications for CTC analyses include real-time non-invasive monitoring of CTCs as biomarkers of either sensitivity or acquired resistance to new cancer therapies, identifying new potential therapeutic targets to directly suppress cancer metastasis. Additionally, as technologies for CTC detection become increasingly sensitive and reliable, it will be important to apply these at earlier stages of cancer progression with the goal of early cancer detection. Although the potential applications of CTC analyses appear extraordinarily promising, the development of appropriate, high-throughput and reliable technological platforms for rare tumor cell detection within blood specimens remains a critical impediment. In fact, appropriate interpretation of many reported molecular analyses of CTCs requires an understanding of the technical limitations of the assays used to make these observations. In the following sections, we review the technologies currently available for CTC isolation and the utility of CTCs as a diagnostic and prognostic marker in various cancers and focus on the molecular properties of these rare cells, which may help define their biology $[34,35]$.

\section{CIRCULATING TUMOR CELL DETECTION TECHNOLOGIES}

The presence of CTCs in an autopsy of a patient who succumbed to advanced metastatic cancer was first reported in 1869. To date, however, only limited information on the numbers of these cells in the blood stream at different stages of cancer and in different types of cancer, their molecular and biological heterogeneity, and their significance in the natural history of the disease is available. In the absence of any gold standard with which to measure various technologies, defining their absolute accuracy, sensitivity, and specificity in detecting CTCs remains a challenge. The ultimate goal (that is; to isolate efficiently this rare population of cells in a viable and intact state and with high purity from the vast number of surrounding blood cells) presents a formidable technological challenge [13]. The molecular characterization of CTCs is considered a real-time 'liquid biopsy' that may contribute to the development of improved individualized therapy and the monitoring of responses to treatment of cancer patients. Hence, a great deal of effort and resources has been invested in developing meth- 
ods for CTC detection [36].

To date, the only U.S. FDA approved technology for CTC detection and quantification with prognostic purposes in metastatic breast, colorectal, or prostate cancer is the CellSearch (Veridex LLC, Raritan, NJ, USA), which utilizes magnetic beads coated with anti-epithelial cell adhesion molecule (EpCAM) antibodies to isolate CTCs [37]. Another antibody-mediated capture technology has been developed, the 'CTC-chip,' which has shown superior detection rates of CTCs of approximately $100 \%$ in metastatic tumors, including lung cancer. The CTC-chip is a microfluidicbased platform capable of separating viable CTCs from peripheral blood samples on the basis of the interaction of target CTCs with EpCAM-coated microposts under laminar flow conditions and without requisite pre-labeling or processing of samples. Despite their usefulness, EpCAM-based methods can have some drawbacks for CTC detection and capture in epithelial tumors, and can be thus limited in their performance by the intrinsic variability of these cells [13].

Indeed, the isolation of CTCs in non-small cell lung cancer (NSCLC) is lower compared to other tumors, and a proportion of NSCLC CTCs can be missed because they undergo epithelial to mesenchymal transition (EMT) and down-regulate their epithelial markers during progression. In addition to CellSearch, other label-free approaches to isolate CTCs that do not rely on the expression of specific cell surface markers but on other inherent properties instead, such as size (i.e., ISET; Rare Cell Diagnostics, Paris, France), deformability, dielectric susceptibility, and/or negative selection of white blood cells, have been developed and tested in solid tumors. It was previously believed that the enumeration of tumor cells in the blood could be used alone as a barometer to measure the level of aggressiveness of a particular cancer; however, improvements in next-generation sequencing (NGS) and isolation methodologies have allowed for analyses of DNA and RNA from isolated cells to gain insight into cancer driver genes. Since singleCTC analyses have provided evidence of genetic heterogeneity at the level of an individual cell, many studies have investigated their diagnostic potential and application in cancer management [21].

\section{CLINICAL RELEVANCE AND BIOLOGICAL IMPLICATIONS}

The detection of CTCs in the peripheral blood of patients with solid epithelial tumors (such as breast, prostate, lung, and colon cancers) holds great promise, and many exciting technologies have been developed over the past several years. In addition, there is a wealth of information on the clinical validation of CTC detection and enumeration (for example, large-scale pooled analysis of thousands of patients with breast cancer) [38], which resulted in the inclusion of CTCs in the new edition of the tumor-node-metastasis cancer staging manual in 2010, as classification cM0. Currently, more than 400 clinical trials use CTCs as biomarkers, and PubMed lists more than 14,000 publications on CTCs. Many publications report patients in advanced stages, but there is also an increasing number of publications on patients at earlier disease stages without clinical and radiologic signs of overt metastases, particularly in breast cancer [39], but also in other tumor entities, such as bladder cancer [40]. These publications show a significant correlation between CTC counts and prognosis, suggesting that CTCs are either surrogates of metastatic activity or are causally involved in the metastatic process [14]. Interestingly, CTCs are observed months and years after surgical resection of the primary tumor [41]. Regarding the short lifespan of CTCs in the circulation [42], this observation suggests that dissociated tumor cells (DTCs) or micrometastases replenish the pool of CTCs over a long period of time [43]. Thus, the analysis of these CTCs may provide unique information about the molecular characteristics of occult minimal residual disease (MRD) in patients with cancer without clinically detectable overt metastases. The detection and molecular analysis of MRD is of the utmost importance for tailoring systemic adjuvant therapy aimed at preventing the progression of DTC/micrometastases to overt metastases but, at present, this decision is usually based on statistical risk assessment and the analysis of a very small sample of the primary tumor $[8,44]$.

\section{POTENTIAL AND CHALLENGES}

Detecting CTCs remains technically challenging. CTCs present at very low concentrations of one tumor cell in a background of millions of blood cells. Their identification and characterization require extremely sensitive and specific analytic methods, which are usually a combination of complex enrichment and detection procedures. In particular, early-stage patients present with a very low concentration of CTCs and may therefore require more sensitive CTC assays and/or analysis of large blood volumes [22].

Nevertheless, recent studies have documented the potential of CTC analyses for in-depth assessments of viable metastatic tumor 
cells at various levels (DNA, RNA, and proteins) and functionalities (in vitro/in vivo) [45], including NGS analyses [46] and xenotransplantation of CTCs into immunodeficient mice [47]. These technologies may contribute to the identification of metastasesinitiator cells; these cells are the prime targets of anti-metastatic therapies and may have developed special resistance mechanisms. At present, more sensitive technologies that also capture CTCs that have undergone EMT, a process linked to cancer stemness and chemotherapy resistance, are being developed $[48,49]$.

Despite the numerous scientific publications related to CTC detection in lung cancer patients, physicians do not use this biomarker in routine clinical practice. This can be explained by the large number of methods available for CTC detection and the difficulty faced by the physician and the biologist in selecting the optimal method for use [50]. In this context, it is noteworthy that the only FDA approved method for CTC detection, the CellSearch method, has been approved for CTC detection in metastatic breast, prostate, and colon cancer patients, but not in metastatic lung cancer patients. This has added some confusion to the use of this indirect technology for lung cancer patient CTC detection. Moreover, since CTCs that have undergone EMT cannot express epithelial biomarkers, the CellSearch system can certainly miss the detection of a subpopulation of CTCs of interest in lung cancer patients. Direct technologies for CTC detection in lung cancer, such as the ISET approach (Rarecells Diagnostics, Paris, France) are certainly highly attractive [39], but the results obtained by different teams should be validated in independent and large multicenter studies. ISET allows for the cytological characterization of CTCs isolated from lung cancer patients according to the classical morphological criteria used by the cytopathologists for distinguishing between benign and malignant epithelial cells [51]. Moreover, this approach allows for an immunocytochemical assessment of CTCs expressing epithelial and/or mesenchymal biomarkers. Other direct technologies, such as those developed by ScreenCell (Sarcelles, France), also allow for the morphological assessment of CTCs in lung cancer patients. Many other methods are currently being developed for CTC characterization, such as a method allowing for the functional evaluation of CTCs and the characterization of a subpopulation of malignant cells [52].

\section{ADVANTAGES OF LIQUID BIOPSY}

Many of the major recent advances in targeted therapies have relied on the acquisition of tumor tissue via biopsy before the initiation of therapy or after the onset of resistance. The availability of tissue for molecular analysis has been instrumental in understanding the primary mechanism of action of agents such as trastuzumab, imatinib, cetuximab, and vemurafenib. Likewise, access to tumor tissue after clinical resistance has helped define mechanisms of secondary resistance to these targeted agents, often in the very pathway or gene involved in their responsiveness $[53,54]$. Although tumor tissue is the gold standard for clinical and investigational sequencing, major barriers exist in terms of acquisition and utility. Biopsies are an inconvenience from a scheduling perspective; they also increase the cost of patient care and are yet another uncomfortable, invasive procedure for patients that often do not influence outcome. Finally, and most importantly, biopsies are not without clinical complications. A review of the investigative biopsies at the MD Anderson Cancer Center reported adverse event rates of $17.1 \%$ and $1.6 \%$ for thoracic biopsies and abdominal/ pelvic sampling, respectively [55].

In addition to the issues related to tissue acquisition, sample preservation and tumor heterogeneity also hamper the use of tumor tissue for cancer sequencing [56]. Most tumor tissue is preserved in formalin-fixed paraffin-embedded (FFPE) blocks, which crosslink DNA and, in some cases, can result in FFPE samples being inadequate for molecular analysis. Although this has improved for individual gene mutations and targeted gene sets, there are still limitations for whole-genome and exome analyses. Furthermore, the quantity of tumor cells in each biopsy varies and is largely dependent on the tumor cellularity (percent tumor) and size of the specimen acquired [57]. This is further compounded by small tissue amounts from fine-needle aspirates or core-needle biopsies, which often result in smaller amounts of tumor tissue for molecular analysis compared with surgically resected specimens. Arguably, the major limitation of tissue biopsy is heterogeneity [58], which characterizes most advanced cancers. Cancers are heterogeneous, with different areas of the same tumor showing different genetic profiles (i.e., intra-tumoral heterogeneity); likewise, heterogeneity exists between metastases within the same patient (i.e., inter-metastatic heterogeneity). A biopsy or tissue section from one part of a solitary tumor will miss the molecular intra-tumoral and inter-metastatic heterogeneity [59,60].

To overcome the limitations of tissue biopsies, less invasive techniques capable of capturing tumor heterogeneity and the molecular changes cancer cells undergo when they are exposed to therapy 
are needed. The measurement of CTCs by liquid biopsy can, noninvasively, provide reliable information regarding prognosis, recurrence, and treatment response, as compared to invasive diagnostic modalities, such as trans-bronchial or fine-needle biopsy and thoracoscopic surgery. CTCs include a variety of subtypes with different functional characteristics. EMT, a process in which epithelial cells convert to mesenchymal cells, contributes substantially to tumor heterogeneity and is crucial to metastatic processes and the acquisition of chemo resistance [61].

A liquid biopsy focusing on the analysis of CTCs and circulating ctDNA in the blood of patients with cancer has received enormous attention because of its obvious clinical implications for personalized medicine. Analyses of CTCs and ctDNA have opened up new diagnostic avenues and are, to date, the cornerstones of liquid biopsy diagnostics [45].

A liquid biopsy can, in principle, provide the same genetic information as a tissue biopsy, which is necessary to interrogate key companion diagnostics [62,63]. The liquid biopsy approach holds clear advantages over 'traditional' tissue biopsies. It is a source of fresh tumor-derived material, unhampered by preservatives. Sampling the blood is minimally invasive and avoids the complications of biopsies [3].

\section{CHALLENGES FOR LIQUID BIOPSY}

In particular, a more mature understanding of the biology behind 'circulating tumor cells' (ctDNA, CTCs, and exosomes) will help us determine whether the molecular profiles generated from these sources truly reflect the physiological disease state of the patient, and whether they can help physicians reliably detect and monitor the disease. To confirm this, we must uncover the origin and dynamics of these tumor parts in the circulation and determine their biological significance and clinical relevance. Although the exact mechanisms behind the release [64] and dynamics of ctDNA remain unknown, several hypotheses exist to explain the existence of tumor DNA in the bloodstream. Perhaps the most widely accepted theory is that tumor cells release DNA via apoptosis, necrosis, or cell secretion in the tumor microenvironment [22]. Alternatively, a patient with an excessive number of CTCs of more than 100,000 was described who, despite having progressive disease, had a low ctDNA allelic frequency in the range of merely 2\%-3\% [65]. While in most patients CTC number and ctDNA levels are mutually correlated, such cases illustrate that exceptions do exist and that the underlying biology of both CTC and ctDNA release is still poorly understood. Other basic unknowns regarding liquid biopsy implementation in the clinic revolve around questions of whether ctDNA actually offers a full representation of a patient's cancer, whether all existing metastases contribute to the ctDNA, CTCs, and exosomes found in the bloodstream, and whether all tumor cells release an equal amount of ctDNA into the circulation. To establish to what extent ctDNA represents metastatic heterogeneity, one study followed a patient with metastatic estrogen receptor-positive and HER2-positive breast cancer over 3 years [66]. However, before liquid biopsies can serve as viable diagnostic assays, pre-analytical steps, such as the collection of bio fluid (such as blood, serum, and plasma), centrifugation settings, isolation reagents, and storage conditions must be standardized to ensure reproducible processing procedures. Furthermore, analytical steps, such as the quantification of ctDNA and subsequent mutational analysis (such as the NGS assay and sequencing platform itself), must be validated to simulate clinical settings. In addition, sensitivities and specificities of the applied assays must be robust, reproducible, and have the appropriate internal and external quality controls [30].

\section{CONCLUSION}

Cancer is a complex, heterogeneic, and dynamic disease involving multiple gene-environment interactions, and affecting numerous biological pathways. As such, the development of reliable and robust non-invasive platforms constitutes a vital step toward realizing the potential of precision medicine. Current work in the liquid biopsy field continues to show great potential utility in the diagnosis and stratification of cancer patients and exemplifies a surrogate method for monitoring treatment response compared to the tissue biopsy approach. The ease and frequency made possible by serial liquid biopsy collection offer plenty of advantages compared to standard surgical procedures, which include, in particular, the opportunity for more rapid course correction when administering therapies. As technological advances continue and further innovations in liquid biopsy methodology emerge in parallel, this approach will hopefully enable methods for the pre-diagnostic assessment of cancer risk. As our knowledge of the biology behind ctDNA improves, so too will the management of cancer patients as the liquid biopsy method becomes a clinical reality. Improvements in the clinical outcomes of many cancer types are 
likely to be achieved by identification of the molecular events that underlie their pathogenesis. With the use of so-called biomarkers, therapies tailored to the genetic composition of tumors can be administered. Tumor genotyping is one possible method of categorizing tumors for clinical decisions and has the potential to identify patients who will be likely to respond to various drugs.

\section{ACKNOWLEDGMENTS}

This work was supported by the grants from the National Cancer Center (1810960-1), Republic of Korea.

\section{REFERENCES}

1. Aravanis AM, Lee M, Klausner RD. Next-generation sequencing of circulating tumor DNA for early cancer detection. Cell 2017;168:571-4.

2. Tang L, Zhao S, Liu W, Parchim NF, Huang J, Tang Y, et al. Diagnostic accuracy of circulating tumor cells detection in gastric cancer: systematic review and meta-analysis. BMC Cancer 2013;13:314.

3. Arneth B. Update on the types and usage of liquid biopsies in the clinical setting: a systematic review. BMC Cancer 2018;18:527.

4. Chu D, Park BH. Liquid biopsy: unlocking the potentials of cell-free DNA. Virchows Arch 2017;471:147-54.

5. Bettegowda C, Sausen M, Leary RJ, Kinde I, Wang Y, Agrawal N, et al. Detection of circulating tumor DNA in early- and late-stage human malignancies. Sci Transl Med 2014;6:224ra24.

6. Marusyk A, Almendro V, Polyak K. Intra-tumour heterogeneity: a looking glass for cancer? Nat Rev Cancer 2012;12:323-34.

7. Heitzer E, Ulz P, Geigl JB. Circulating tumor DNA as a liquid biopsy for cancer. Clin Chem 2015;61:112-23.

8. Tamimi Y, Gupta I, Al-Moundhri M, Burney I. Micrometastatic circulating tumor cells; a challenge for an early detection and better survival rates. J Carcinog Mutagen 2015;6:4. https://doi.org/10.4172/2157-2518.1000229.

9. Hanahan D, Weinberg RA. Hallmarks of cancer: the next generation. Cell 2011;144:646-74.

10. Carmichael A, Sami AS, Dixon JM. Breast cancer risk among the survivors of atomic bomb and patients exposed to therapeutic ionising radiation. Eur J Surg Oncol 2003;29:475-9.

11. Lianidou ES. Circulating tumor cells: new challenges ahead. Clin Chem 2012;58:805-7.

12. Racila E, Euhus D, Weiss AJ, Rao C, McConnell J, Terstappen LW, et al. Detection and characterization of carcinoma cells in the blood. Proc Natl Acad Sci U S A 1998;95:4589-94.

13. Andree KC, van Dalum G, Terstappen LW. Challenges in circulating tumor cell detection by the CellSearch system. Mol Oncol 2016;10:395-407.

14. Hayes DF, Cristofanilli M, Budd GT, Ellis MJ, Stopeck A, Miller MC, et al. Circulating tumor cells at each follow-up time point during therapy of metastatic breast cancer patients predict progression-free and overall survival. Clin Cancer Res 2006;12(14 Pt 1):4218-24.

15. Kowalik A, Kowalewska M, Gozdz S. Current approaches for avoiding the limitations of circulating tumor cells detection methods-implications for diagnosis and treatment of patients with solid tumors. Transl Res 2017; 185:58-84

16. Hwang WL, Hwang KL, Miyamoto DT. The promise of circulating tumor cells for precision cancer therapy. Biomark Med 2016;10:1269-85.

17. Onstenk W, de Klaver W, de Wit R, Lolkema M, Foekens J, Sleijfer S. The use of circulating tumor cells in guiding treatment decisions for patients with metastatic castration-resistant prostate cancer. Cancer Treat Rev 2016;46:42-50.

18. Pestrin M, Salvianti F, Galardi F, De Luca F, Turner N, Malorni L, et al. Heterogeneity of PIK3CA mutational status at the single cell level in circulating tumor cells from metastatic breast cancer patients. Mol Oncol 2015;9:749-57.

19. Alix-Panabieres C, Pantel K. Clinical applications of circulating tumor cells and circulating tumor DNA as liquid biopsy. Cancer Discov 2016;6: 479-91.

20. Galletti G, Worroll D, Nanus DM, Giannakakou P. Using circulating tumor cells to advance precision medicine in prostate cancer. J Cancer Metastasis Treat 2017;3:190-205.

21. Heitzer E, Auer M, Gasch C, Pichler M, Ulz P, Hoffmann EM, et al. Complex tumor genomes inferred from single circulating tumor cells by arrayCGH and next-generation sequencing. Cancer Res 2013;73:2965-75.

22. Riethdorf S, Fritsche H, Muller V, Rau T, Schindlbeck C, Rack B, et al. Detection of circulating tumor cells in peripheral blood of patients with metastatic breast cancer: a validation study of the CellSearch system. Clin Cancer Res 2007;13:920-8.

23. Lorente D, Mateo J, de Bono JS. Molecular characterization and clinical utility of circulating tumor cells in the treatment of prostate cancer. Am Soc Clin Oncol Educ Book 2014:e197-203. https://doi.org/10.14694/EdBook_AM.2014.34.e197.

24. Dotan E, Cohen SJ, Alpaugh KR, Meropol NJ. Circulating tumor cells: evolving evidence and future challenges. Oncologist 2009;14:1070-82.

25. Valastyan S, Weinberg RA. Tumor metastasis: molecular insights and evolving paradigms. Cell 2011;147:275-92.

26. Klein CA. Parallel progression of primary tumours and metastases. Nat Rev Cancer 2009;9:302-12.

27. Turajlic S, Swanton C. Metastasis as an evolutionary process. Science 2016; 352:169-75.

28. Navin N, Kendall J, Troge J, Andrews P, Rodgers L, McIndoo J, et al. Tumour evolution inferred by single-cell sequencing. Nature. 2011;472:90-4.

29. Navin NE. The first five years of single-cell cancer genomics and beyond. Genome Res 2015;25:1499-507.

30. Alix-Panabieres C, Pantel K. Circulating tumor cells: liquid biopsy of cancer. Clin Chem 2013;59:110-8.

31. Aceto N, Bardia A, Miyamoto DT, Donaldson MC, Wittner BS, Spencer JA, et al. Circulating tumor cell clusters are oligoclonal precursors of breast cancer metastasis. Cell 2014;158:1110-22.

32. Miyamoto DT, Zheng Y, Wittner BS, Lee RJ, Zhu H, Broderick KT, et al. RNA-Seq of single prostate CTCs implicates noncanonical Wnt signaling in antiandrogen resistance. Science 2015;349:1351-6.

33. Hartkopf AD, Wagner P, Wallwiener D, Fehm T, Rothmund R. Changing levels of circulating tumor cells in monitoring chemotherapy response in patients with metastatic breast cancer. Anticancer Res 2011;31:979-84.

34. Kitz J, Lowes LE, Goodale D, Allan AL. Circulating tumor cell analysis in preclinical mouse models of metastasis. Diagnostics (Basel) 2018;8:E30. https://doi.org/10.3390/diagnostics8020030.

35. Gonzalez-Angulo AM, Hennessy BT, Mills GB. Future of personalized medicine in oncology: a systems biology approach. J Clin Oncol 2010;28: 2777-83.

36. Coumans FA, Ligthart ST, Uhr JW, Terstappen LW. Challenges in the enumeration and phenotyping of CTC. Clin Cancer Res 2012;18:5711-8.

37. Grover PK, Cummins AG, Price TJ, Roberts-Thomson IC, Hardingham JE. Circulating tumour cells: the evolving concept and the inadequacy of 
their enrichment by EpCAM-based methodology for basic and clinical cancer research. Ann Oncol 2014;25:1506-16.

38. Sasportas LS, Hori SS, Pratx G, Gambhir SS. Detection and quantitation of circulating tumor cell dynamics by bioluminescence imaging in an orthotopic mammary carcinoma model. PLoS One 2014;9:e105079.

39. Ried K, Eng P, Sali A. Screening for circulating tumour cells allows early detection of cancer and monitoring of treatment effectiveness: an observational study. Asian Pac J Cancer Prev 2017;18:2275-85.

40. Rink M, Chun FK, Dahlem R, Soave A, Minner S, Hansen J, et al. Prognostic role and HER2 expression of circulating tumor cells in peripheral blood of patients prior to radical cystectomy: a prospective study. Eur Urol 2012;61:810-7.

41. Budd GT, Cristofanilli M, Ellis MJ, Stopeck A, Borden E, Miller MC, et al. Circulating tumor cells versus imaging: predicting overall survival in metastatic breast cancer. Clin Cancer Res 2006;12:6403-9.

42. Cohen SJ, Punt CJ, Iannotti N, Saidman BH, Sabbath KD, Gabrail NY, et al. Prognostic significance of circulating tumor cells in patients with metastatic colorectal cancer. Ann Oncol 2009;20:1223-9.

43. Bork U, Rahbari NN, Scholch S, Reissfelder C, Kahlert C, Buchler MW, et al. Circulating tumour cells and outcome in non-metastatic colorectal cancer: a prospective study. Br J Cancer 2015;112:1306-13.

44. Muller V, Stahmann N, Riethdorf S, Rau T, Zabel T, Goetz A, et al. Circulating tumor cells in breast cancer: correlation to bone marrow micrometastases, heterogeneous response to systemic therapy and low proliferative activity. Clin Cancer Res 2005;11:3678-85.

45. Pantel K, Alix-Panabieres C. Real-time liquid biopsy in cancer patients: fact or fiction? Cancer Res 2013;73:6384-8.

46. Yee SS, Lieberman DB, Blanchard T, Rader J, Zhao J, Troxel AB, et al. A novel approach for next-generation sequencing of circulating tumor cells. Mol Genet Genomic Med 2016;4:395-406.

47. Cristofanilli M, Budd GT, Ellis MJ, Stopeck A, Matera J, Miller MC, et al. Circulating tumor cells, disease progression, and survival in metastatic breast cancer. N Engl J Med 2004;351:781-91.

48. Tsai JH, Yang J. Epithelial-mesenchymal plasticity in carcinoma metastasis. Genes Dev 2013;27:2192-206.

49. Pierga JY, Hajage D, Bachelot T, Delaloge S, Brain E, Campone M, et al. High independent prognostic and predictive value of circulating tumor cells compared with serum tumor markers in a large prospective trial in first-line chemotherapy for metastatic breast cancer patients. Ann Oncol 2012;23:618-24.

50. Gorges TM, Tinhofer I, Drosch M, Rose L, Zollner TM, Krahn T, et al. Circulating tumour cells escape from EpCAM-based detection due to epithelial-to-mesenchymal transition. BMC Cancer 2012;12:178.

51. Hofman VJ, Ilie MI, Bonnetaud C, Selva E, Long E, Molina T, et al. Cytopathologic detection of circulating tumor cells using the isolation by size of epithelial tumor cell method: promises and pitfalls. Am J Clin Pathol
2011;135:146-56

52. Yao X, Choudhury AD, Yamanaka YJ, Adalsteinsson VA, Gierahn TM, Williamson CA, et al. Functional analysis of single cells identifies a rare subset of circulating tumor cells with malignant traits. Integr Biol (Camb) 2014;6:388-98.

53. Misale S, Yaeger R, Hobor S, Scala E, Janakiraman M, Liska D, et al. Emergence of KRAS mutations and acquired resistance to anti-EGFR therapy in colorectal cancer. Nature 2012;486:532-6.

54. Rosa R, Monteleone F, Zambrano N, Bianco R. In vitro and in vivo models for analysis of resistance to anticancer molecular therapies. Curr Med Chem 2014;21:1595-606.

55. Giuliano M, Giordano A, Jackson S, Hess KR, De Giorgi U, Mego M, et al. Circulating tumor cells as prognostic and predictive markers in metastatic breast cancer patients receiving first-line systemic treatment. Breast Cancer Res 2011;13:R67.

56. Shyr D, Liu Q. Next generation sequencing in cancer research and clinical application. Biol Proced Online 2013;15:4.

57. Travis WD, Brambilla E, Noguchi M, Nicholson AG, Geisinger KR, Yatabe $\mathrm{Y}$, et al. International association for the study of lung cancer/american thoracic society/european respiratory society international multidisciplinary classification of lung adenocarcinoma. J Thorac Oncol 2011;6: 244-85.

58. Cheema PK, Menjak IB, Winterton-Perks Z, Raphael S, Cheng SY, Verma $S$, et al. Impact of reflex EGFR/ ALK testing on time to treatment of patients with advanced nonsquamous non-small-cell lung cancer. J Oncol Pract 2017;13:e130-8.

59. Nowell PC. The clonal evolution of tumor cell populations. Science 1976; 194:23-8.

60. Llovet JM, Zucman-Rossi J, Pikarsky E, Sangro B, Schwartz M, Sherman M, et al. Hepatocellular carcinoma. Nat Rev Dis Primers 2016;2:16018.

61. Powell AA, Talasaz AH, Zhang H, Coram MA, Reddy A, Deng G, et al. Single cell profiling of circulating tumor cells: transcriptional heterogeneity and diversity from breast cancer cell lines. PLoS One 2012;7:e33788.

62. Santarpia M, Liguori A, D’Aveni A, Karachaliou N, Gonzalez-Cao M, Daffina MG, et al. Liquid biopsy for lung cancer early detection. J Thorac Dis 2018;10(Suppl 7):S882-97.

63. Cree IA. Liquid biopsy for cancer patients: principles and practice. Pathogenesis 2015;2:1-4.

64. Pantel K, Alix-Panabières C. Liquid biopsy: potential and challenges. Mol Oncol 2016;10:371-3.

65. Rapisuwon S, Vietsch EE, Wellstein A. Circulating biomarkers to monitor cancer progression and treatment. Comput Struct Biotechnol J 2016; 14:211-22.

66. Wang R, Li X, Zhang H, Wang K, He J. Cell-free circulating tumor DNA analysis for breast cancer and its clinical utilization as a biomarker. Oncotarget 2017;8:75742-55. 